\title{
200-Gb/s Polarization Multiplexed Doubly Differential QPSK Signal Transmission over 80-km SSMF Using Tandem SSB without Optical Amplification
}

\author{
Tingting Zhang, Christian Sanchez, lan Phillips, Stylianos Sygletos, Andrew Ellis
}

Aston Institute of Photonic Technologies (AIPT), Aston University, Birmingham, B4 7ET, UK, zhangt16@aston.ac.uk

\begin{abstract}
We propose 200-Gb/s polarization multiplexed tandem SSB DDQPSK with intradyne detection for $80-\mathrm{km}$ SSMF transmission. Without optical amplification, dispersion compensation or carrier recovery, the simulated receiver sensitivity for $80-\mathrm{km}$ transmission was below $-25.5 \mathrm{dBm}$ (at $7 \% \mathrm{HD}$ FEC threshold).
\end{abstract}

\section{Introduction}

The recent commercialization of the $100-\mathrm{Gb} / \mathrm{s}$ technology has motivated research on higher capacity optical interfaces for the next generation Ethernet. Increasing the bit rate per optical carrier is regarded as the effective solution to reduce overall cost of optical system ${ }^{1}$. The proposed approaches include single carrier coupled with high-order modulation format ${ }^{2}$ or high symbol rate $^{3}$. However, high-speed and high-resolution analogue/digital converters, large-bandwidth optoelectronic components and powerful digital signal processing (DSP) are required to ensure good system performance. Other schemes like coherent optical orthogonal frequency division multiplexing ${ }^{4}$ are similarly reliant on DSP and the system performance is very sensitivity to the symbol timing ${ }^{5}$ and carrier frequency offset (FO). For long-haul applications, optical system without optical amplification is preferred to minimise cost, footprint and power consumption.

In this paper, we propose and numerically demonstrate $80-\mathrm{km}$ standard single mode fibre (SSMF) transmission using a 200-Gb/s doubly differential quadrature phase shift keying (DDQPSK) signal with tandem single sideband ${ }^{6}$ (TSSB) modulation and polarization division multiplexing (PDM). The system operates without optical amplification, digital dispersion compensation or carrier recovery. Compared with double sideband and single sideband (SSB) modulation, TSSB makes the best use of the bandwidth, and doubles the system capacity with independent signals carried on the upper sideband (USB) and lower sideband (LSB). Combining TSSB with subcarrier multiplexing ${ }^{7}$ (SCM) provides adequate dispersion tolerance, allowing the avoidance of dispersion compensation. Similarly, doubly differential (DD) encoding $^{8}$ eliminates the requirement of carrier recovery including FO estimation and phase noise compensation, greatly simplifying the DSP.
Numerical results demonstrate that FOs within \pm $2.3 \mathrm{GHz}$, caused by the optical local oscillator (OLO) (within $\pm 2 \mathrm{GHz}$ ) and RF local oscillators (RLOs) (within $\pm 300 \mathrm{MHz}$ ) in the receiver, have negligible impact on the back-to-back (B2B) performance with a maximum $0.16-\mathrm{dB} Q$ factor variation. The receiver sensitivity at $7 \%$ harddecision forward error correction (HD-FEC) threshold of $3.8 \mathrm{E}-3$ is below $-25.5 \mathrm{dBm}$, giving a 20-dB power budget and less than 0.2-dB transmission penalty.

\section{Simulation setup}

As shown in Fig. 1, for each subcarrier and polarization, two independent $2^{7}-1$ pseudo random binary sequences (PRBSs) with the same length of 32768 were firstly bit wise Graycoded into QPSK signals and then doubly differentially pre-coded before being up-sampled to 16 Samples/symbol. Square root raised cosine (SRRC) filters with 0.3 roll-off factor and bandwidth matched at the symbol rate were applied in the transmitter for pulse shaping. Three RLOs $(13 \mathrm{GHz}, 26 \mathrm{GHz}$ and $39 \mathrm{GHz}$ ), which could be generated from a single oscillator using frequency multipliers, were employed for signal up-conversion via ideal IQ mixers before the passive combiner. The combined SCM-DDQPSK signals (one for the USB and the other for the LSB) were separately sent to the input port 1 and port 2 of a four-port 90 degree hybrid (known as a branchline combiner) with the coupled signal and its Hilbert transform achieved at port 3 and 4 . The output signals of two such 90 degree hybrids (one for each polarization) were uploaded to VPI TransmissionMaker V9.7 via cosimulation with Matlab to drive a dual-polarization (DP) IQ modulator with half-wave voltage of $5 \mathrm{~V}$ and $6-\mathrm{dB}$ insertion loss, giving the generated PDM-TSSB signal with an output power of $-5.6 \mathrm{dBm}$.

A $100-\mathrm{kHz}$ linewidth laser emitting at 193.1 $\mathrm{THz}$ with $10-\mathrm{dBm}$ output power was used in the transmitter, whilst a free-running laser with 2.9- 


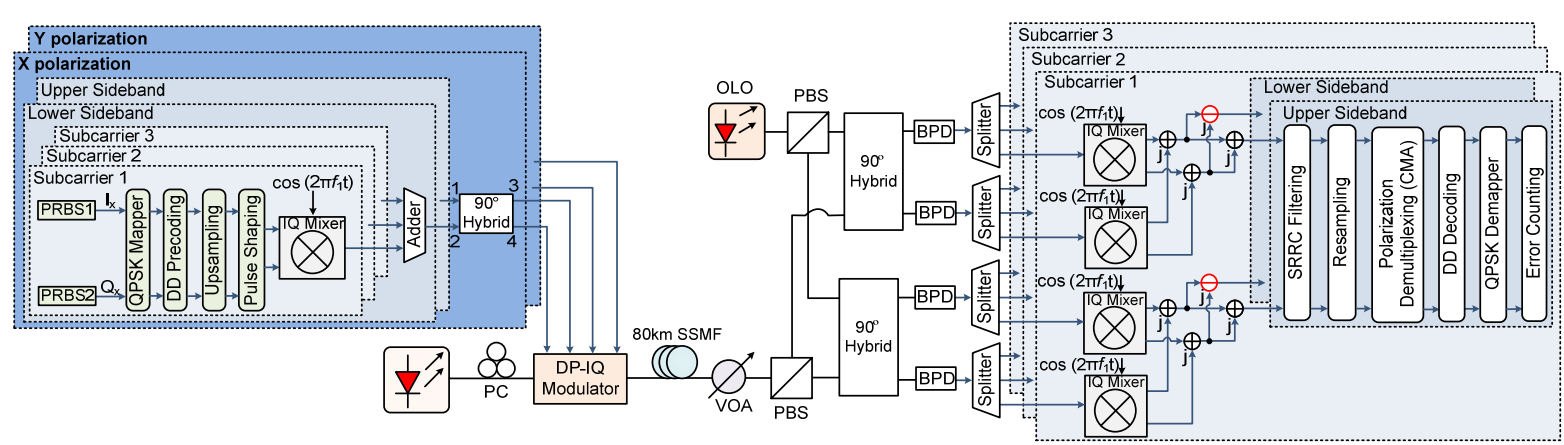

Fig. 1 Simulation setup of the proposed 200-Gb/s PDM-DDQPSK based TSSB system. PC: polarization controller; VOA: variable optical attenuator.

$\mathrm{MHz}$ linewidth and $18-\mathrm{dBm}$ output power was used as the OLO in the receiver. The intradyne receiver also included two polarization beam splitters (PBSs), two 90 degree optical hybrids, and four 43-GHz balanced photodetectors (BPDs) with 0.54-A/W responsivity, shot noise and $40-p A / \sqrt{ } \mathrm{Hz}$ thermal noise current. The detected real and imaginary components for both polarizations were down-converted using IQ mixers with the LSB and the USB information separately obtained utilizing linear power combiners (as depicted in Fig. 1). After being filtered by the SRRC low-pass filters $(0.3$ roll-off factor, 3-dB bandwidth of $14.3 \mathrm{GHz}$ ), the signals were down-sampled to 2 Samples/symbol. 7-tap adaptive finite impulse response filters with tap weights optimized through constant modulus algorithm were employed in a butterfly configuration for polarization de-multiplexing of the quasi baseband signals. After DD decoding and constellation de-mapping, the final error counting was implemented and summed over all subcarriers and polarizations.

\section{Results and discussion}

The frequency and phase mismatch between the lasers and RLOs in the transceiver will contribute to the final FO and overall phase noise, respectively. Since typical tunable laser sources may be readily tuned to be within $2 \mathrm{GHz}$ of a target wavelength, thus the OLO-induced FOs were set to be within $\pm 2 \mathrm{GHz}$ in simulation. Moreover, the RLOs' frequency may not be exactly the same as that in the transmitter, thus the FO caused by the first RLO was set to be within $\pm 100 \mathrm{MHz}$, leading to a maximum FO of $\pm 300 \mathrm{MHz}$ for the third RLO because of the frequency multipliers. The impact of the total FOs on the 200-Gb/s PDM-DDQPSK B2B system was first evaluated at a received optical power (ROP) of $-22.9 \mathrm{dBm}$. As shown in Fig. 2, the B2B performance remains practically constant along the studied FOs with a maximum $0.16-\mathrm{dB} Q$ factor variation. This indicates that $D D$ encoding can tolerate the overall FOs within $\pm 2.3 \mathrm{GHz}$, regardless of the contributed source. Here, the $Q$ factor is accumulated according to the formula: $Q=20 \log _{10}\left(\sqrt{2} \operatorname{erfc}^{-1}(2 B E R)\right)$ where erfc ${ }^{-1}$ represents the inverse of the complementary error function and the bit error rate (BER) is averaged by running the simulation ten times. Note that in the remainder of our paper, for simplification, only the OLO-induced FOs were considered and the same RLOs were employed in the receiver. Fig. 3 shows the B2B performance for both SSB (only LSB or USB signal transmitted) and TSSB with the proposed intradyne detection. It can be seen that TSSB technique enables the achievement of doubled

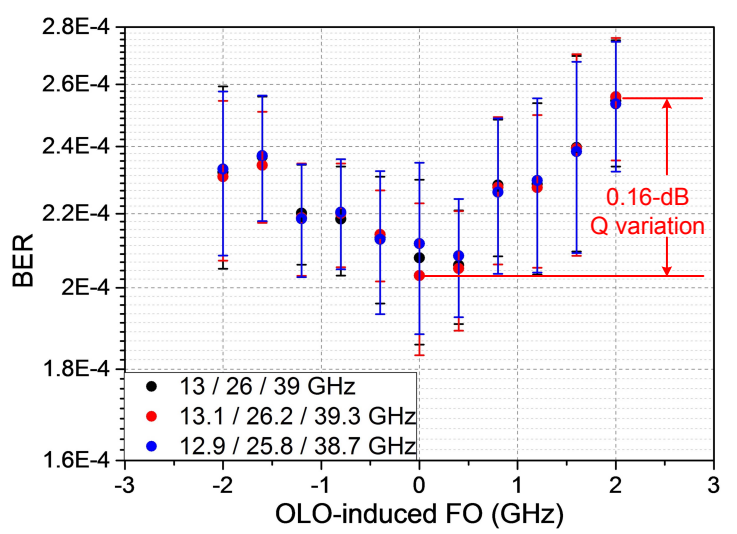

Fig. 2 B2B performance of the 200-Gb/s PDM-DDQPSK system using different RLOs (shown in legend) in the receiver with various OLO-induced FOs at -22.9-dBm ROP.

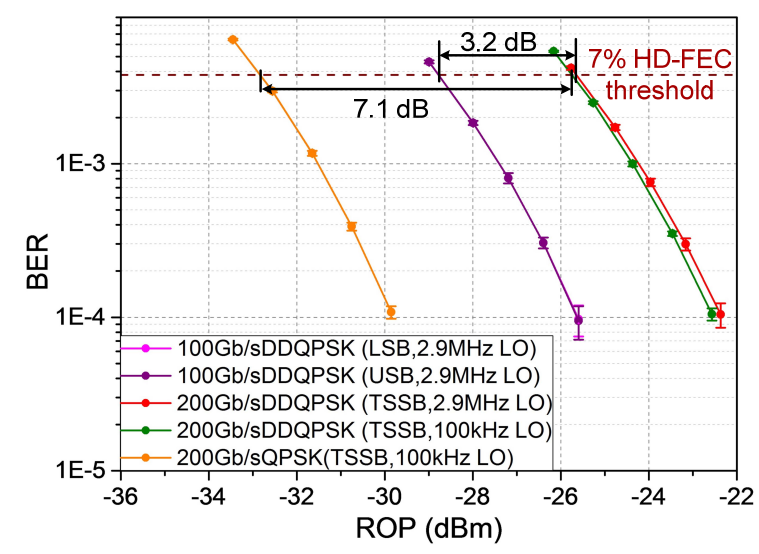

Fig. 3 B2B performance of the PDM-DDQPSK system using SSB/TSSB modulation and $2.9-\mathrm{MHz} / 100-\mathrm{kHz} \mathrm{LO}$, and the TSSB PDM-QPSK system using 100-kHz linewidth LO without FO. 


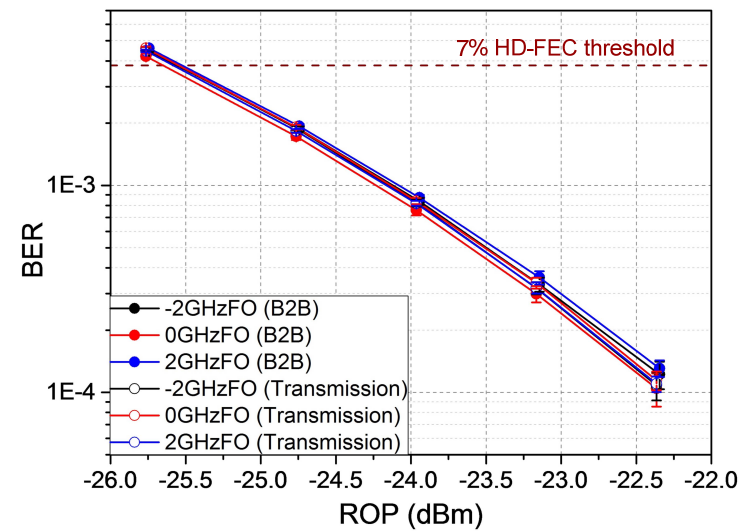

Fig. 4 Performance of the 200-Gb/s PDM-DDQPSK B2B and 80-km SSMF transmission system with different FOs.

capacity $(200 \mathrm{~Gb} / \mathrm{s})$ at the cost of a $3.2-\mathrm{dB}$ receiver sensitivity degradation. This penalty is close to the theoretical value $(3 \mathrm{~dB})$ owing to doubled number of channels in the TSSB system. The additional $0.2-\mathrm{dB}$ penalty is attributed to the crosstalk induced by the residual sideband of the LSB signal on the USB counterpart, and vice versa. To investigate the system tolerance to the phase noise, we employed LOs with different linewidth (100 kHz and $2.9 \mathrm{MHz}$ ) for comparison. Around $0.1-\mathrm{dB}$ penalty is observed in Fig. 3 for the 200-Gb/s PDM-DDQPSK system using 2.9$\mathrm{MHz}$ linewidth OLO. This small degradation indicates high robustness to the phase noise, brought about by DD encoding. In practice, the linewidth tolerance would be shared by the lasers used in the transceivers. Compared with the PDM-QPSK system with full carrier recovery, the PDM-DDQPSK system suffers an expected receiver sensitivity offset of $7.1 \mathrm{~dB}$ (theoretically $7.17 \mathrm{~dB}^{9}$ ) owing to enhanced noise power induced by the two differential operations.

Fig. 4 compares the performance for the 200$\mathrm{Gb} / \mathrm{s}$ PDM-DDQPSK signal B2B and 80-km SSMF transmission under different FOs with a 2.9-MHz linewidth OLO used in the receiver. Even without carrier recovery, the system performance for both cases are almost immune to the FOs with less than $0.2-\mathrm{dB}$ sensitivity penalty at $7 \%$ HD-FEC threshold, demonstrating the advantage of DD coding. Without dispersion compensation, the receiver sensitivity for $80-\mathrm{km}$ transmission at BER of $3.8 \mathrm{E}-3$ is below -25.5 $\mathrm{dBm}$, which is degraded by less than $0.2 \mathrm{~dB}$ in comparison with the B2B case.

\section{Conclusion}

We have numerically demonstrated a $200-\mathrm{Gb} / \mathrm{s}$ TSSB modulated DDQPSK signal transmission over 80-km SSMF without optical amplification, dispersion compensation or carrier recovery. Thanks to DD encoding, the impact of the overall FOs induced by the OLO and RLOs on the B2B performance has been minimized with only 0.16 -
$\mathrm{dB} Q$ factor variation. Therefore, no carrier recovery for both optical and RF local oscillators is required in our system. High robustness to the phase noise, brought about by DD encoding, has also greatly relaxed the linewidth requirement on the OLOs utilized in coherent receivers. In the case of a $2.9-\mathrm{MHz}$ linewidth OLO, the receiver sensitivity (at BER of $3.8 \mathrm{E}-3$ ) for the $200-\mathrm{Gb} / \mathrm{s}$ DDQPSK signal after 80-km SSMF transmission was below $-25.5 \mathrm{dBm}$ with less than $0.2-\mathrm{dB}$ transmission penalty.

\section{Acknowledgements}

This work is partially funded by the Engineering and Physical Sciences Research Council projects PEACE (EP/L000091/1), UPON (EP/M005283/1), SPFS (EP/L00044X/1), TOM3 (EP/M009092/1), UNLOC (EP/J017582/1), and by the European Union project INVENTION (659950). The data underlying this publication can be found at http://doi.org/10.17036/researchdata.aston.ac.uk.000 00239.

\section{References}

[1] P. Winzer, "Beyond 100G ethernet," IEEE Commun. Mag., Vol. 48, no. 7, p. 26-30 (2010).

[2] H. Chien et al., "On Single-Carrier 400G Line Side Optics Using PM-256QAM," Proc. ECOC, Dusseldorf (2016).

[3] P. Winzer et al., "Generation and 1,200-km transmission of 448-Gb/s ETDM 56-Gbaud PDM 16-QAM using a single $\mathrm{I} / \mathrm{Q}$ modulator," Proc. ECOC, PDP2.2, Torino (2010).

[4] X. Liu et al., "448-Gb/s Reduced-Guard-Interval COOFDM Transmission Over 2000 km of Ultra-Large-Area Fiber and Five 80-GHz-Grid ROADMs," J. Lightwave Technol., Vol. 29, no.4, p.483-490 (2011).

[5] Z. Dong et al., "Bandwidth-Efficient Modulation for Hybrid 10G/100G Optical Communication Networks," E Photon. Technol. Lett., Vol. 28, no. 4, p. 469-472 (2016).

[6] A. Narasimha et al., "Tandem single sideband modulation scheme for doubling spectral efficiency of analogue fibre links," Electron. Lett., Vol. 36, no. 13, p. 1135-1136 (2000).

[7] F. A. Gutiérrez et al., "All-Analogue Real-Time Broadband Filter Bank Multicarrier Optical Communications System," J. Lightwave Technol., Vol. 33, no. 24, p. 5073-5083 (2015).

[8] A. J. Walsh et al., "Reduced OSNR penalty for frequency drift tolerant coherent packet switched systems using doubly differential decoding," Proc. OFC, Th4D.8, San Francisco (2014).

[9] T. Zhang et al., "A High-sensitivity Coherent Receiver without Frequency Recovery Enabled by Doubly Differential QPSK," Proc. CLEO, STu3M, San Jose (2017). 